\title{
Spectral Variability Signatures of Relativistic Shocks in Blazars
}

\author{
Markus Böttcher* \\ Centre for Space Research, North-West University, Potchefstroom, 2520, South Africa \\ E-mail: Markus.Bottcher@nwu.ac.za

\section{Matthew G. Baring} \\ Department of Physics and Astronomy, Rice University, Houston, TX 77005-1892, USA \\ E-mail: baring@rice.edu
}

\begin{abstract}
Mildly relativistic, oblique shocks are frequently invoked as possible sites of relativistic particle acceleration and production of strongly variable, polarized multi-wavelength emission from relativistic jet sources such as blazars, via diffusive shock acceleration (DSA). In recent work, we had self-consistently coupled DSA and radiation transfer simulations in blazar jets. These one-zone models determined that the observed spectral energy distributions (SEDs) of blazars strongly constrain the nature of the hydromagnetic turbulence responsible for pitch-angle scattering. In this paper, we expand our previous work by including full time dependence and treating two emission zones, one being the site of acceleration. This modeling is applied to a multiwavelength flare of the flat spectrum radio quasar 3C 279, fitting snap-shot SEDs and light curves. We predict spectral hysteresis patterns in various energy bands as well as cross-band time lags with optical and $\mathrm{GeV} \gamma$-rays as well as radio and X-rays tracing each other closely with zero time lag, but radio and X-rays lagging behind the optical and $\gamma$-ray variability by several hours.
\end{abstract}

6th Annual Conference on High Energy Astrophysics in Southern Africa

1 - 3 August, 2018

Parys, South Africa

${ }^{*}$ Speaker. 


\section{Introduction}

Relativistic, oblique shocks have long been considered as one of the leading contenders for the sites of relativistic particle acceleration in relativistic jet sources, such as blazars and gamma-ray bursts, resulting in the observed rapidly variable, often highly polarized multi-wavelength (MW) emission. The dominant particle acceleration mechanism at such shocks is referred to as diffusive shock acceleration (DSA). Particle acceleration results from repeated shock crossings of particles gyrating along large-scale ordered magnetic fields. The reversal of particle momenta $p$ along magnetic field lines is facilitated by diffusive pitch-angle scatterings (PAS). Several theoretical studies of particle acceleration at relativistic shocks (e.g., [18, 13, 14, 25]) have shown that this process can result in a wide variety of spectral indices. Such studies of the particle acceleration mechanism, however, usually do not consider the resulting radiative signatures in a self-consistent manner.

On the other hand, models focusing on the multi-zone radiative transfer problem for internalshock models of blazars (e.g., $[19,24,22,20,23,15,7,17,10,11])$ do not typically address the details of particle acceleration, but assume an ad-hoc injection of purely non-thermal relativistic particles, usually with a truncated power-law distribution in energy. In recent work [3], we coupled the Monte Carlo (MC) simulations of DSA of Summerlin \& Baring [25] with radiative transfer routines of Böttcher et al. [8]. This provided, for the first time, a consistent description of the DSA process and its radiative signatures in mildly relativistic, oblique shocks in blazar jets. Fits to spectral energy distributions (SEDs) of three blazars indicated the need for a strongly energydependent PAS diffusive mean-free path $\lambda_{\text {pas }} \propto p^{\alpha}$, with $\alpha \sim 2-3$ required, depending on the type of blazar considered. This may be considered as evidence of hydromagnetic turbulence levels gradually decreasing with increasing distance from the shock $[3,9]$.

In this work, we present an extension of the DSA + radiaton-transfer model of [3], including full time variability. We thus make predictions for time-dependent snap-shot SEDs and multiwavelength light curves which can be further analyzed to predict multi-wavelength spectral hysteresis patterns and inter-band time lags. In Section 2, we describe our model setup and the numerical scheme we developed for simulating time-dependent DSA and radiation transfer in internal shocks in blazars. The application to a multi-wavelength flare of 3C 279 is presented in Section 3, yielding fairly good MW spectral fits and distinctive temporal characteristics.

\section{Setup and Numerical Scheme}

Our time-dependent shock-acceleration and radiation-transfer simulations are based on the premise that a DSA-type particle acceleration mechanism is at work in the high-energy emission region of a blazar jet at all times. A quiescent state is established through a balance between time-independent DSA in a small acceleration zone and radiative cooling and escape of particles in a larger radiation zone of length $\ell_{\text {rad }}$, which is identified with the high-energy emission region, as described in detail in [3]. Variability arises from the passage of a mildly relativistic shock through the density and magnetic field structures in the high-energy emission region, nominally on an observed time scale $\Delta t_{\mathrm{obs}}=\left(\ell_{\mathrm{rad}} / v_{s}\right)(1+z) / \delta$. Here $v_{s}$ is the shock velocity in the co-moving frame of the jet material, $z$ is the cosmological redshift of the source, and $\delta$ is the Doppler factor arising from the bulk motion of the jet material with respect to the observer. 
The thermal + non-thermal particle distributions resulting from DSA have been evaluated using the Monte Carlo (MC) code of [25]. In the DSA scenario, the Fermi-I acceleration process that includes episodes of shock drift energization is facilitated by stochastic PAS of charges spiraling along magnetic field lines. PAS is parameterized through the corresponding mean-free path $\lambda_{\text {pas }}$ as an energy-dependent multiple $\eta(p)$ of the particle's gyro radius, $r_{g}=p c /(q B)$, where $p$ is the particle's momentum, such that $\lambda_{\text {pas }}=\eta(p) r_{g}$. The energy dependence of the mean-free-path parameter $\eta$ is defined as a power-law in the particle's momentum, $\eta(p)=\eta_{1} p^{\alpha-1}$, so that $\lambda_{\text {pas }} \propto p^{\alpha}$ and $\eta_{1}$ describes the mean free path in the non-relativistic limit, $\gamma \rightarrow 1$.

The MC simulations of [25] illustrate that DSA leads to a non-thermal power-law tail of relativistic particles which have been accelerated out of the remaining thermal pool. A high-energy cut-off $\left(\gamma_{\max }\right)$ of the non-thermal particle spectra results from the balance of the acceleration time scale $t_{\text {acc }}\left(\gamma_{\max }\right)=\eta\left(\gamma_{\max }\right) t_{\mathrm{gyr}}\left(\gamma_{\max }\right)$ with the radiative energy loss time scale. If synchrotron losses dominate, $\gamma_{\max } \propto B^{-1 / 2}$. This will lead to a synchrotron peak energy $E_{\mathrm{sy}} \sim 240 \delta \eta^{-1}\left(\gamma_{\max }\right) \mathrm{MeV}$. Notably, this synchrotron peak energy is independent of the magnetic field $B$, as $E_{\mathrm{sy}} \propto B \gamma_{\max }^{2}$. Blazars typically show synchrotron peaks in the IR to soft X-rays. In order to reproduce these, the pitch angle scattering mean-free-path parameter $\eta\left(\gamma_{\max }\right)$ has to assume values of $\sim 10^{4}-10^{8}$. However, [25] have shown that $\eta_{1}$ must be significantly smaller than this value in order to obtain efficient injection of particles out of the thermal pool into the non-thermal acceleration process. From these arguments we can infer that $\eta(\gamma)$ must be strongly energy dependent [3].

The DSA-generated thermal + non-thermal electron spectra serve as a particle injection term into simulations of subsequent radiative cooling of the electrons. As relevant radiative mechanisms, synchrotron radiation in a tangled magnetic field, synchrotron self-Compton (SSC) radiation, and Compton scattering of external radiation fields (external Compton $=\mathrm{EC}$ ) on various plausible target photon fields are taken into account in our simulations. Particles may also leave the emission region on a time scale parameterized as a multiple of the light-crossing time scale of the emission region, $t_{\text {esc }, \mathrm{e}}=\eta_{\text {esc }} \ell_{\text {rad }} / c$. Figure 1 shows the energy dependence of the relevant time scales for the steady state generated to describe the quiescent-state multi-wavelength emission of 3C 279 (see Section 3). DSA will be effective up to an energy $\gamma_{\max }$, where the radiative cooling time scale becomes shorter than the acceleration time scale. Figure 1 shows that for almost all particles at lower energies, $\gamma<\gamma_{\max }$, the acceleration time scale is many orders of magnitude shorter than the radiative cooling and/or or escape timescales. Thus, numerically, DSA may be well represented as instantaneous injection of relativistic particles at a (time-dependent) rate $Q_{e}\left(\gamma_{e}, t\right)\left[\mathrm{cm}^{-3} \mathrm{~s}^{-1}\right]$, which is then followed by evolution on the radiative and escape time scales.

The evolution of the electron distribution is simulated by numerically solving a Fokker-Planck equation of the form

$$
\frac{\partial n_{e}\left(\gamma_{e}, t\right)}{\partial t}=-\frac{\partial}{\partial \gamma_{e}}\left(\dot{\gamma}_{e} n_{e}\left[\gamma_{e}, t\right]\right)-\frac{n_{e}\left(\gamma_{e}, t\right)}{t_{\mathrm{esc}, \mathrm{e}}}+Q_{e}\left(\gamma_{e}, t\right)
$$

using an implicit scheme as described in [6]. Here, $\dot{\gamma}_{e}$ represents the combined radiative energy loss rate of the electrons, and all quantities are in the co-moving frame of the emission region. Radiation transfer is being handled by forward evolution of a continuity equation for the photons,

$$
\frac{\partial n_{\mathrm{ph}}(\varepsilon, t)}{\partial t}=\frac{4 \pi j_{\varepsilon}}{\varepsilon m_{e} c^{2}}-c \kappa_{\varepsilon} n_{\mathrm{ph}}(\varepsilon, t)-\frac{n_{\mathrm{ph}}(\varepsilon, t)}{t_{\mathrm{esc}, \mathrm{ph}}},
$$


where $J_{\varepsilon}$ and $\kappa_{\varepsilon}$ are the emissivity and absorption coefficient, respectively, $\varepsilon=h v /\left(m_{e} c^{2}\right)$ is the dimensionless photon energy, and $t_{\mathrm{esc}, \mathrm{ph}}$ is the photon escape time scale, $t_{\mathrm{esc}, \mathrm{ph}}=(4 / 3) \ell_{\mathrm{rad}} / \mathrm{c}$ for a spherical geometry [4]. Radiative processes are evaluated using the routines of [8]. The observed flux is provided by the escaping photons, such that

$$
v F_{v}^{\mathrm{obs}}\left(v_{\mathrm{obs}}, t_{\mathrm{obs}}\right)=\frac{\varepsilon^{2} m_{e} c^{2} n_{\mathrm{ph}}(\varepsilon, t) \delta^{4} V_{\mathrm{rad}}}{4 \pi d_{L}^{2}(1+z) t_{\mathrm{esc}, \mathrm{ph}}}
$$

where $\varepsilon=(1+z) \varepsilon_{\mathrm{obs}} / \delta$ and $V_{\mathrm{rad}} \approx(4 / 3) \pi \ell_{\mathrm{rad}}^{3}$ is the co-moving volume of the emission region, and jet-frame and observer time intervals are related through $\Delta t_{\mathrm{obs}}=\Delta t(1+z) / \delta$.

Our code produces snap-shot SEDs and multi-wavelength light curves at pre-specified frequencies. It also extracts local spectral indices at the same frequencies for each time step, for the purpose of plotting hardness-intensity diagrams. Correlations between the light curves at different frequencies and possible inter-band time lags are evaluated using the Discrete Correlation Function analysis [12]. For each flare simulation, we first let the code run until it reaches a stable equilibrium with the quiescent-state parameters. An individual flaring event is then simulated by changing various input parameters with a step function in time for the duration $\Delta t=\ell_{\mathrm{rad}} / v_{s}$.
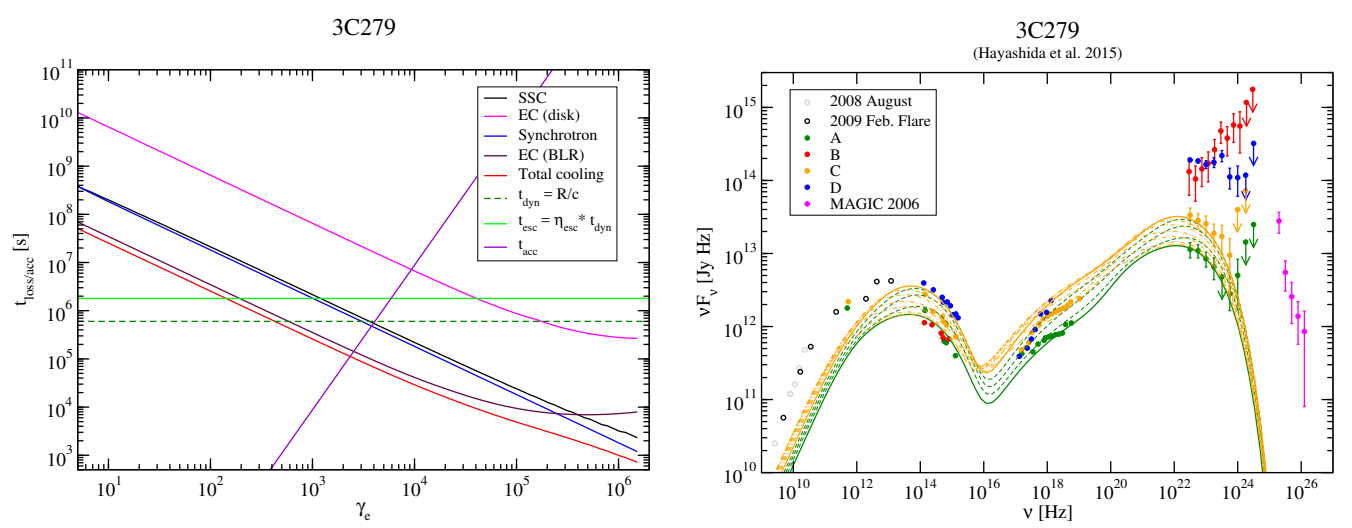

Figure 1: Left panel: Relevant acceleration (purple), radiative cooling (black, pink, blue, brown, red see legend), dynamical (green dashed) and escape (light green) time scales for electrons in the simulated quiescent-state equilibrium configuration for 3C 279 (see Section 3). Right panel: Snap-shot SEDs of 3C279 during 2013 - 2014. Data are from [16]. Green curves illustrate the spectral evolution during the rising part of the simulated Flare C; yellow curves show the evolution during the decaying part. See text for details.

\section{Application to 3C 279}

As an application of our code, we provide a fit to a multi-wavelength flare of the well-known Flat Spectrum Radio Quasar 3C 279. Hayashida et al. [16] identified several individual flaring episodes in 2013 - 2014. For the purpose of this study we select Flare C, which showed simultaneous flaring in the optical, X-ray, and $\gamma$-rays and may therefore well be represented by an increase in the particle injection luminosity, plausibly caused by an internal shock in the jet of 3C 279. The characteristic time scale of short-term flares of 3C 279 during the $2013-2014$ period (includng Flare C) is $\Delta t_{\mathrm{obs}} \sim 1$ day. With a typical Doppler factor of $\delta=15$, a redshift of 
Table 1: Parameters for the model fit to 3C 279.

\begin{tabular}{cc}
\hline Parameter & Value \\
\hline Quiescent electron injection luminosity & $L_{\text {inj,qu }}=1.1 \times 10^{43} \mathrm{erg} \mathrm{s}^{-1}$ \\
Flaring electron injection luminosity & $L_{\text {inj,fl }}=5.0 \times 10^{43} \mathrm{erg} \mathrm{s}^{-1}$ \\
Emission region size & $\ell_{\text {rad }}=1.8 \times 10^{16} \mathrm{~cm}$ \\
Jet-frame magnetic field & $B=0.65 \mathrm{G}$ \\
Escape time scale parameter & $\eta_{\mathrm{esc}}=3$ \\
Pitch-angle scattering m.f.p. parameter & $\eta_{1}=100$ \\
Pitch-angle scattering m.f.p. scaling index & $\alpha=3$ \\
Bulk Lorentz factor & $\Gamma=\delta=15$ \\
Accretion-disk luminosity & $L_{d}=6 \times 10^{45} \mathrm{erg} \mathrm{s}^{-1}$ \\
Distance of active region from BH & $z_{i}=0.1 \mathrm{pc}^{-3}$ \\
External radiation field energy density & $u_{\mathrm{ext}}=4 \times 10^{-4} \mathrm{erg} \mathrm{cm}^{-3}$ \\
External radiation field BB temperature & $T_{\mathrm{ext}}=300 \mathrm{~K} .^{2}$
\end{tabular}

$z=0.536$, and a mildly relativistic shock with $v_{s} \sim 0.7 \mathrm{c}$, this implies a size of the active region of $\ell_{\mathrm{rad}} \sim 1.8 \times 10^{16} \mathrm{~cm}$. We assume a viewing angle of $\theta_{\mathrm{obs}} \approx 1 / \Gamma$, so that $\delta \approx \Gamma=15$.

Both the direct accretion-disk radiation field and an isotropic external radiation field are needed to model the $\gamma$-ray spectrum of $3 \mathrm{C} 279$. The latter is assumed to be dominated by the dust-torus radiation field, which is approximated as a thermal blackbody at a temperature of $T_{\mathrm{ext}}=300 \mathrm{~K}$. The most relevant parameters are listed in Table 1. The quiescent state fit is illustrated by the solid green line in Fig. 1. We find that it can be well described with an electron injection spectrum produced by DSA with a pitch-angle scattering mean free path scaling as $\lambda_{\text {pas }}=100 r_{g} p^{2}$, i.e., $\lambda_{\text {pas }} \propto p^{3}$. Based on the competition of acceleration and cooling time scales, as illustrated in Fig. 1, electrons are accelerated up to a maximum energy of $\gamma_{\max }=2.4 \times 10^{3}$.
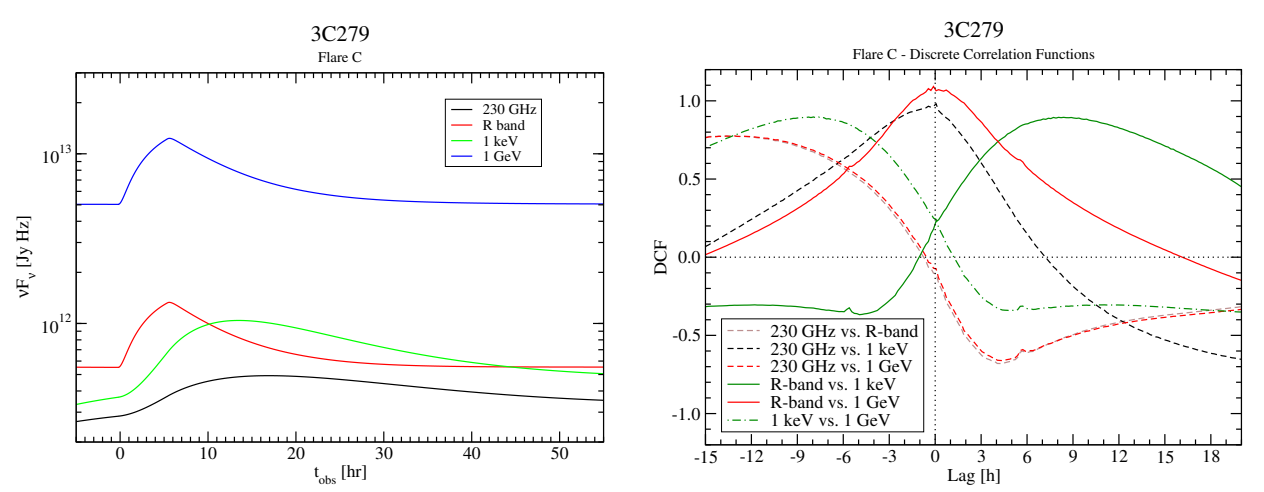

Figure 2: Left: Multi-wavelength light curves extracted from the simulation illustrated in Fig. 1. Right: Discrete cross correlation functions evaluated from the light curves shown in the left panel.

For the evolutionary model of flare $\mathrm{C}$, the only parameter changed to produce the flare is the electron injection luminosity, corresponding to a larger number of electrons accelerated per unit 
time, without changing the characteristics of the acceleration process. The green curves in Fig. 1 show SEDs during the rising phase of the flare, the solid yellow curve indicates the snap-shot SED during the peak of flare $\mathrm{C}$, while the remaining yellow curves illustrate the decaying part of the flare. The resulting light curves in the mm radio, optical, $\mathrm{X}$-ray and $\mathrm{GeV} \gamma$-ray bands are illustrated in the left panel of Fig. 2, while cross-correlations are shown in the right panel of Fig. 2.

The model predicts, as expected, that the optical and $\gamma$-ray light curves are closely correlated with zero time lag, as those bands are produced by synchrotron and Compton emission from electrons of similar energies and therefore comparable cooling times. The X-ray emission is expected to lag behind the optical and $\gamma$-ray emissions by $\sim 8 \mathrm{hr}$, while the mm radio band is expected to show an even longer delay behind optical and $\gamma$-rays, with slightly weaker correlation. Unfortunately, the light curve coverage in existing data (including those for 3C 279 in [16]) is not sufficient for a meaningful comparison of our predictions with data.

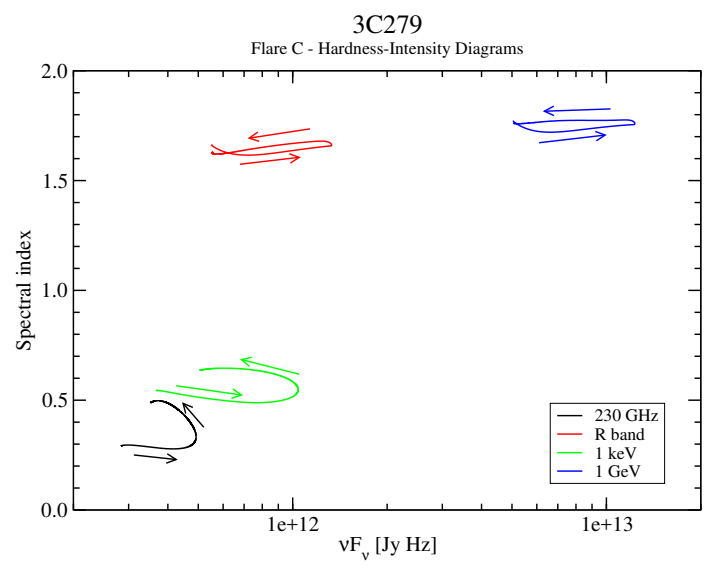

Figure 3: Hardness-intensity diagrams extracted from the same flare simulation illustrated in Figs. 1 and 2.

Figure 3 shows the hardness-intensity diagrams extracted from our simulatons to flare C. While only very weak spectral variability is predicted in the optical and $\mathrm{GeV} \gamma$-ray bands, pronounced counter-clockwise spectral hysteresis (harder rising-flux spectra; softer decaying-flux spectra) is expected in the mm radio and X-ray bands. Such spectral hysteresis has so far only been clearly identified in the X-ray spectra of high-frequency peaked BL Lac objects (e.g., [26]), where the Xray emission is synchrotron-dominated. Observing such features in other wavelength bands would enable stringent constraints on the magnetic field in the emission region (see, e.g., [5]). Such a goal is quite challenging in the $\mathrm{GeV}$ band because of limited count statistics in Fermi-LAT observations.

Summary: We have presented the first time-dependent coupled DSA + radiation-transfer simulations, based on the MC simulations of DSA by [25] and the electron dynamics and radiation transfer modules of $[6,8]$. This has been applied to a specific multi-wavelength flare of 3C 279, namely Flare $\mathrm{C}$ of [16], which was selected because of its approximately equal flare amplitudes in the optical, X-rays, and GeV $\gamma$-rays. The evolving spectra can be well modeled by assuming that the shock passage through an active region in the jet affects primarily the number of electrons accelerated via DSA. The model predicts well-correlated variability in the optical and $\mathrm{GeV} \gamma$-ray bands and between $\mathrm{mm}$ radio and $\mathrm{X}$-ray bands. The $\mathrm{mm}$ radio and $\mathrm{X}$-ray band variability also is well correlated with the optical and $\gamma$-rays, but lags behind those variations by $\sim 8-10$ hours. Spectral 
variability in the optical and $\gamma$-ray bands is expected to be weak, but significant counter-clockwise spectral hysteresis is expected in the mm radio and $\mathrm{X}$-ray bands.

Acknowledgements: The work of M. Böttcher is supported through the South African Research Chairs Initiative (grant no. 64789) of the Department of Science and Technology and the National Research Foundation ${ }^{1}$ of South Africa. M. Baring is grateful to NASA for support under the Fermi Guest Investigator Program through grant 80NSSC18K1711.

\section{References}

[1] Abdo, A. A., et al., 2011, ApJ, 727, 129

[2] Ackermann, M., et al., 2012, ApJ, 751, 159

[3] Baring, M. G., Böttcher, M., \& Summerlin, E. J., 2017, MNRAS, 464, 4875

[4] Böttcher, M., Mause, H., \& Schlickeiser, R., 1997, A\&A, 324, 395

[5] Böttcher, M., et al., 2003, ApJ, 596, 847

[6] Böttcher, M., \& Chiang, J., 2002, ApJ, 581, 127

[7] Böttcher, M., \& Dermer, C. D., 2010, ApJ, 711, 445

[8] Böttcher, M., Reimer, A., Sweeney, K., \& Prakash, A., 2013, ApJ, 768, 54

[9] Böttcher, M., Baring, M. G., \& Summerlin, E. J., 2017, in Proc. of "HEASA 2016", PoS, 275, 74

[10] Chen, X., Fossati, G., Liang, E. P., \& Böttcher, M., 2011, MNRAS, 416, 2368

[11] Chen, X., Fossati, G., Böttcher, M., \& Liang, E. P., 2012, MNRAS, 424, 789

[12] Edelson, R. A., \& Krolik, J. H., 1988, ApJ, 333, 646

[13] Ellison, D. C., Reynolds, S. P., \& Jones, F. C., 1990, ApJ, 360, 702

[14] Ellison, D. C., \& Double, G. P., 2004, Astropart. Phys., 22, 323

[15] Graff, P. B., Georganopoulos, M., Perlman, E. S., \& Kazanas, D., 2008, ApJ, 689, 68

[16] Hayashida, M., et al., 2015, ApJ, 807, 79

[17] Joshi, M., \& Böttcher, M., 2011, ApJ, 727, 21

[18] Kirk, J. G., \& Heavens, A. F., 1989, MNRAS, 239, 995

[19] Marscher, A. P., \& Gear, W. K., 1985, ApJ, 298, 114

[20] Mimica, P., Aloy, M. A., Müller, E., \& Brinkmann, W., 2004, A\&A, 418, 947

[21] Sikora, M., Madejski, G., Moderski, R., \& Poutanen, J., 1997, ApJ, 484, 108

[22] Sokolov, A., Marscher, A. P., \& McHardy, I. M., 2004, ApJ, 613, 725

[23] Sokolov, A., \& Marscher, A. P., 2005, ApJ, 625, 52

[24] Spada, M., Ghisellini, G., Lazzati, D., \& Celotti, A., 2001, MNRAS, 325, 1559

[25] Summerlin, E. J., \& Baring, M. G., 2012, ApJ, 745, 63

[26] Takahashi, T., et al., 1996, ApJ, 470, L89

\footnotetext{
${ }^{1}$ Any opinion, finding and conclusion or recommendation expressed in this material is that of the authors and the NRF does not accept any liability in this regard.
} 